\title{
Efficient Modeling of Laser-Plasma Accelerators with INF\&RNO
}

\author{
C. Benedetti, C. B. Schroeder, E. Esarey, C. G. R. Geddes and W. P. Leemans \\ Lawrence Berketey Nattonat Laboratory, Berketey, CA, 94720, USA
}

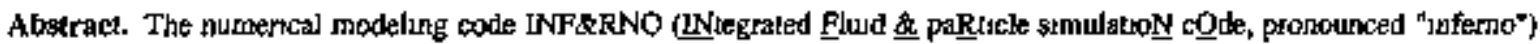
is presented INF\&RNO ws an efficient $2 \mathrm{D}$ cylindrical code to model the interaction of a shont laser pulge with ant underdense plasma The code ts based on an envelope model for the laser while eilher a PIC or a fiud description can be used for the piasma The effect of the laser puise on the plasma is modieled with the imme-ayeraged poderomotwe force These and other feadures allow for a spetdup of 2-4 ofders of magnitude compared to standard full PIC bimulationts while stıll retaining physical fideluty The code has been benchmarked agsunst analytical solutions and 3D PIC sumulatione and here a set of yaladation Itats together with a discussion of the perfomances are presenied
\end{abstract}

Kaywords: laser plestme accelenakos, stmulation, benchmatking

PACS: $5238 \mathrm{Kd}, 47 \mathrm{I}$ ]

\section{INTRODUCTION}

Detatled and reliable numencal modeling in 3D of a laser-plasma acceleralot (LPA) [1, 2], where a short and intenst laser palse interacts with an underdense plasma over dastances ranging from a few millumeters/centumeters (yrelding $\sim 0 \mathrm{~J} / \mathrm{GeV}$ electron energy [3-5]) up to a meter (expected $\sim 10 \mathrm{GeV}$ electrons [6]), is a formutably challenging task A 3D "full" ( $\mathrm{e}$, where we take into account the fastest tirne scale represented by the oscillations of the laser field) particle-nn-cell (PIC) smulation requures $10^{4}-10^{5}$ CPU hours in today's supercompulers for a millmeterscaie plasma and $O\left(10^{6}\right) \mathrm{CPU}$ hours for a centrmeter-scale plasma Since in general the computational conplexity for a fixed accuracy in the results grows more than linearly with the number of time steps, we easily deduce that a simulauon of a meter-scale plasma requires tens of millons of CPU hours and so becomes unfeasible with standard simulation tools [7] However, simulations are requured, since the phystes involyed in the laser-plasma interachon is highly nonlunear and, consequently, abalytical solutions are lacking Numerical modeling plays a central role in belping our understanding of the physics Two solutions have betn proposed to overcome this Iimitation and allow for the sumulation of mulu-GeV LPA stages 1 run the full PIC stmulation in an optimal boosted Lorentz frame [8] instead of $m$ the laboratory frame, $a$ use reduced models The first optron is certanly attractive and strongly pursued by several groups The adventage of running a simulation in a boosted Lorentz frame reles on the fact that, if backward propagatıng waves ( $\mathrm{g}$. Raman backsctttenng) can be neglected, and this ts usually truc grven the phenomenology of LPAs, then it has been sbown [8] that the unbalance between the maximum and mistmum physical scales involved in a simulation, which contribute to set the computational complexity of the problem, is not unvanant under Lorentz transformation it turns out that in general the laboratory frame is not the optimal chose to wu the simulation while running it in a boosted frime can considerably reduce the scale unbalance, shortening (also by several orders of magnutude) the simulation length Applications, estimated computational speedips, and limitations of this techrique are discussed in [8-12] Codes based on reduced models on the other hand allow for a signıicant speedup compared to full PIC simulations etther because of dimensionality reduction $(e g, 2 \mathrm{D}$ cylindrical unstead of full 3D cartesian) or because of approximations in the physical description of the system (e g , quasi-static unstead of fully dynamic plasma response, ponderomotrve approximation unstead of full lorenz force, etc) Even if they may lack important elements of the physics (e $\mathrm{g}$, a quasi-stalic cole can not describe self-injection), their use has been proven to be successful in several relevant scenarios [13- 17$]$

The INF\&RNO computational framework, currenlly under development at LBNL, is a 2D cylundncal (r-z) code that adopts an envelope model for the laser pulse and makes use of the ponderomotve force approxımation to descnte the interaction of the laser pulse with the plasma The plestra can be modeled using ether a PIC or a fuud descnption and its responge is fully dynamic even though a quasi-static module will be avalable soon Both PIC and fluid modaluties are integrated in the same computational framework allowing for staged stmulations (c g, PIC-mode for tijectuon and 
fluid-mode for acceleration). It is also possible to load and track self consistently externally injected bunches. The ultimate goal of the INF\&RNO project is obtaining a fast rectuced code suitable for modeling the relevant features of a LPA producing 10 GeV in a meter-scale plasma (e.g., BELLA [6]) where, for a given problem, it is possible to switch between several physical descriptions'levels of approximations in order to clearly identify in each sibuation the relevant physics involved. In this paper we provide an overview of the INF\&RNO framework (first section) together with a set of validation tests (second section) and a discussion of the performance and the future developments of the code (conclusion and putlook).

\section{THE CODE: NUMERICS AND FEATURES}

JNF\&RNO is a 2D-cylindrical (r-z) code which adopts non-dimensional, "comoving" variables defined as $\xi=$ $k_{p}(z-c t)$ (longitudinal) and $\rho=k_{p} r$ (transverse), where $k_{p}=\omega_{p} / c, \omega_{D}$ is the plasma frequency corresponding to the chosen reference density $n_{0}$, and $c$ is the speed of light. The time is also rescaled with $1 / \omega_{p}$, that is $\tau=\omega_{p} t$. The laser pulse is described using an envelope model [18]. Denoting by $a_{\perp}=e A_{L} / m c^{2}$ the romatized yector potential of the



$$
\left(i \frac{k_{0}}{k_{p}}+\partial_{\xi}\right) \partial_{\tau} \hat{a}=\frac{1}{2} \frac{\delta}{\gamma_{\text {flud }}} \hat{a}-\frac{1}{2} \nabla_{\perp}^{2} a_{1}
$$

where $2 \pi / \ell_{0}$ is the central laser wavelength, $\delta=n / n_{0}$ is the (normalized) plasma density and $\gamma_{\text {nud }}$ is the relativistic factor associated with the local plasma fluid velocity (see below). The fully electronagnetic wakefield is described by the fields $E_{2}, E_{r}, B_{\phi}$ normalized to $E_{0}=m c \omega_{p} / e$, where $n$ and $e$ are respectively mass and ctarge of the electron. The wakefield evolves according to Ampere-Maxwell laws which read

$$
\frac{\partial E_{r}}{\partial \tau}=\frac{\partial\left(E_{r}-B_{\phi}\right)}{\partial \xi}-j_{r}, \frac{\partial E_{t}}{\partial \tau}=\frac{\partial E_{t}}{\partial \xi}+\frac{1}{\rho} \frac{\partial\left(\rho B_{\varphi}\right)}{\partial \rho}-j_{r}, \frac{\partial B_{\phi}}{\partial \tau}=-\frac{\partial\left(E_{r}-B_{\phi}\right)}{\partial \xi}+\frac{\partial E_{z}}{\partial \rho},
$$

where $\left(j_{r}, j_{2}\right)$ are the components of the (normalized) current densily. The background plasma can be modeled using either a PIC or a fluid description while for external injected bunches only the PIC description is currently available. Laser-malter coupling is described via the ponderonotive approximation. The evolution equations for the PIC and fluid modalities are

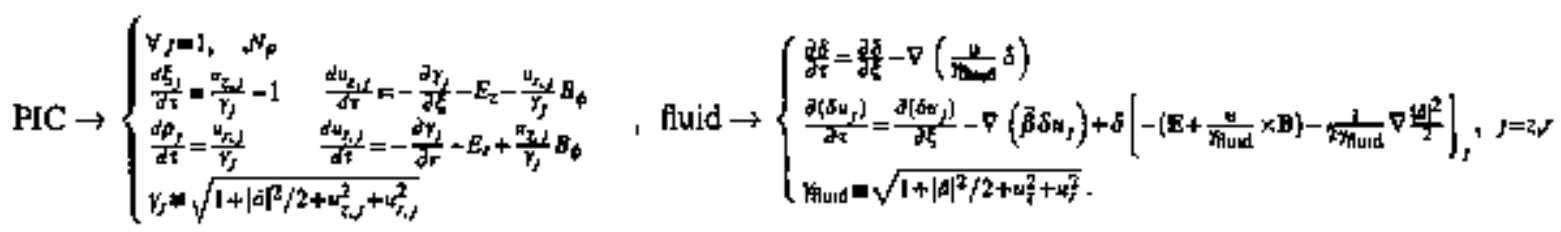

In the PIC part $\left(\xi_{j_{1}} \rho_{,} u_{\xi_{1}, r_{r}} u_{r_{1}}\right)$ are the phase-space coordinales (position and normalized momentum) of the $f^{h}$ numerical particle representing one of the characteristics of the Vlasov equalion for the plasma. In the fuid part the fields $\delta$ and $\mathbf{u}=\left(u_{z_{1}}, w_{1}\right)$ are the plasma density and momentum. The two modalities are integrated in the same computational framework, enabling an essy switch from one description to the other (combined simulations). A ID "full" fuid version of the code is also available.

Concerning numerical aspects, all the fields are discretized into the same 2D mesh (no staggering is adopled). Longitudinal derivatives are computed using a second-order finite difference upwind scheme [19]: $\left(\partial_{\xi} /\right)_{1, s}=\left(-3 h_{y}+\right.$ $\left.4 f_{t+1, j}-f_{t+2, f}\right) /\left(2 A_{\xi}\right)$, where $f_{N,}$ is the field value at the $(i, j)$ node and $A_{\xi}$ the longiludinal cell size. Radial derivatives are computed using a stantard centered second-order accurate scheme. The possibility to adopt a ronunifom radial grid (stretched radial grid) is currently under testing. No singularity exists at the $r=0$ boundary, and from symmetry properties we have $\left.\partial_{\rho} E_{z}\right|_{\rho=0}=\left.E_{\gamma}\right|_{\rho=0}=\left.B_{\phi}\right|_{p=0}=0$ and $\lim _{\rho \rightarrow 0} B_{\phi} / \rho=\partial B_{\phi} /\left.\partial \rho\right|_{\rho=0}$. Second and foutth order Range-Kutta integrators (RK2/RK4) are available for field evolution while plasma particles and externally injected bunches can be pushed with either RK4 or the standard Boris pusher [7]. The implementation of an implicit integration scheme is underway and is related to the inclusion of a quasi-static module for the wakefied and the plasma. Concerning force interpolation and charge/cursent deposition, both linear and quadratic shape functions have been implemented. Compart low-pass filters [20] are available for current and feld smoothing. The user has large freedom 

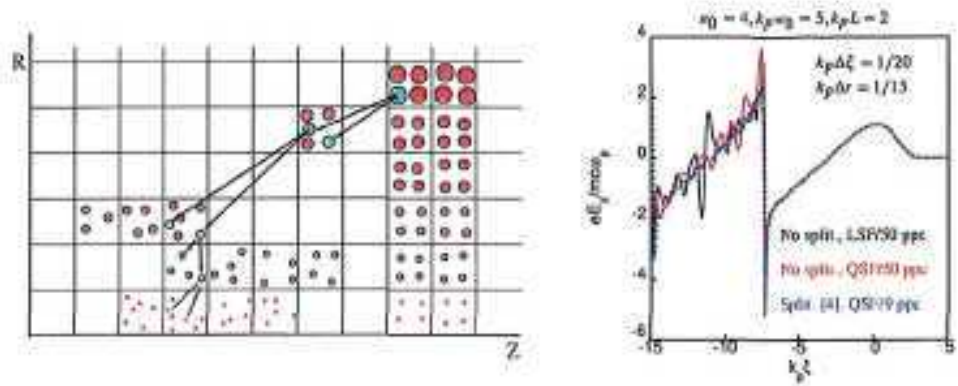

FIGURB 1. Left: particle splitting ptocedure for a "heavy" particle (the blue one) approaching the $r=0$ axis. Right: longitudinal wakefield with and without splitting. Black: without splitting, 50 particles/cell and linear shape function. Red: without splitting, 50 particles/cell and quadratic shape function. Blue: with splitting ( 4 fragments), 9 particles/cell and quadratic shape function.
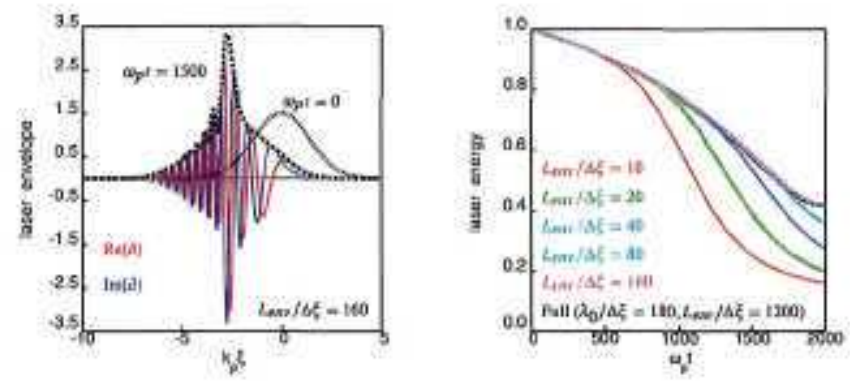

FIGURE 2. ID fluid-envelope simulation: $a_{0}=1.5, k_{0} / k_{p}=20, k_{p} L=2$. Left: black plots are the laser envelope profile at $\omega_{p} t=0$ and $\omega_{p} t=1500$, the red/blue plots are the real/imaginary part of $a$ at $\omega_{p} t=1500$. Right: laser energy evolution deep into depletion as a function of the longitudinal resolution in the envelope (colored plots). The black plot is a full fluid simulation (no envelope approximation) at very high resolution ( 180 points/ $\left.\lambda_{\text {lasef }}\right)$ and can be considered an "exact" result.

in loading numerical particles over the computational domain (the numerical particle distribution is controlled by a simple user-defined routine) and this freedom can be used to selectively provide a better sampling of the plasma phase space distribution within the dynamically interesting zones without greatly increasing the overall number of simulated particles. Because of the cylindrical symmetry, particles loaded at large radii carry generally more charge than particles loaded on-axis. If/when these "heavy" particles approach the $r=0$ axis, they may induce "spikes" in density and currents increasing the noise level in the fields. In INF\&RNO, to partially compensate this detrimental effect, particles with high charge approaching the axis are split into smaller fragments (see Fig. 1 (left)). The method is simple but quite effective as can be seen in Fig. 1 (right). Drawbacks of this approach are a small violation of the local charge/energy conservation and a local heating of the plasma while total charge and momentum are conserved.

We end this section with a comment on the validity of the envelope description when the laser is strongly depleted. Within the envelope approximation there is no need, in principle, to resolve the laser wavelength since the envelope length $\left(L_{\text {env }}\right)$ is the smallest relevant scale as far as the pulse is concerned. However, during nonlinear laser evolution (redshifting, depletion) structures smaller than $L_{\text {env }}$ arise (see Fig. 2 (left)) and the mesh resolution must be high enough to capture them. Fig. 2 (right) shows the evolution, deep into depletion, of the laser energy computed using the envelope model at various longitudinal resolutions (see figure caption for details). As long as the resolution is high enough, the envelope model correctly reproduces the physics still ensuring an effective speed-up (larger than $\sim 2$ ) compared to a "full" description of the laser. The computational savings from implementation of the envelope model will be reduced if modeling deep into depletion is required.

\section{VALIDATION TESTS AND BENCHMARKS}

Test 1 . We consider the diffraction in vacuum (up to 10 Rayleigh lengths) of a tightly focused Gaussian pulse ( $k_{0} w_{0}=20, k_{0} L=40$, where $w_{0}$ and $L$ are respectively the pulse waist and length): the goal is to test the accuracy of the laser envelope solver. In Fig. 3 (left) we show the evolution of the laser vector potential, $a(z) / a(z=0)$, as a 

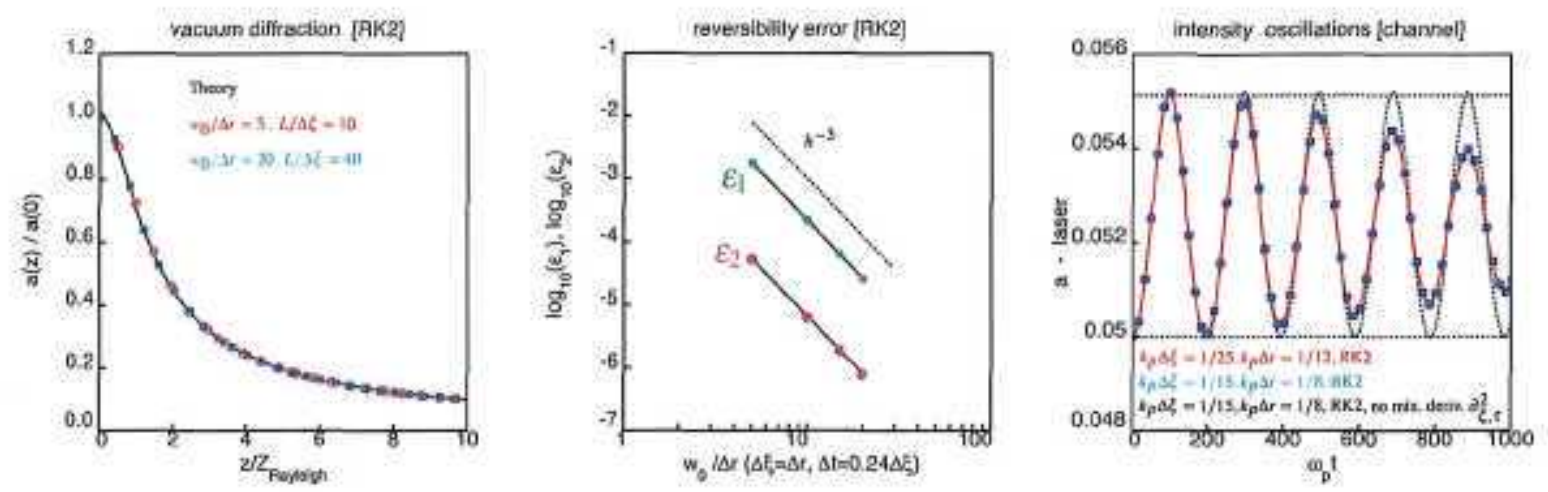

FIGURE 3. Left: vacuum diffraction of a focused laser pulse (see subsection "Test 1 " for details). Center: time reversibility test for the vacuum diffracting pulse changing the resolution (see subsection "Test 1"). Right: mismatch oscillations of a low intensity pulse in a plasma channel (see subsection "Test 2 " for details).
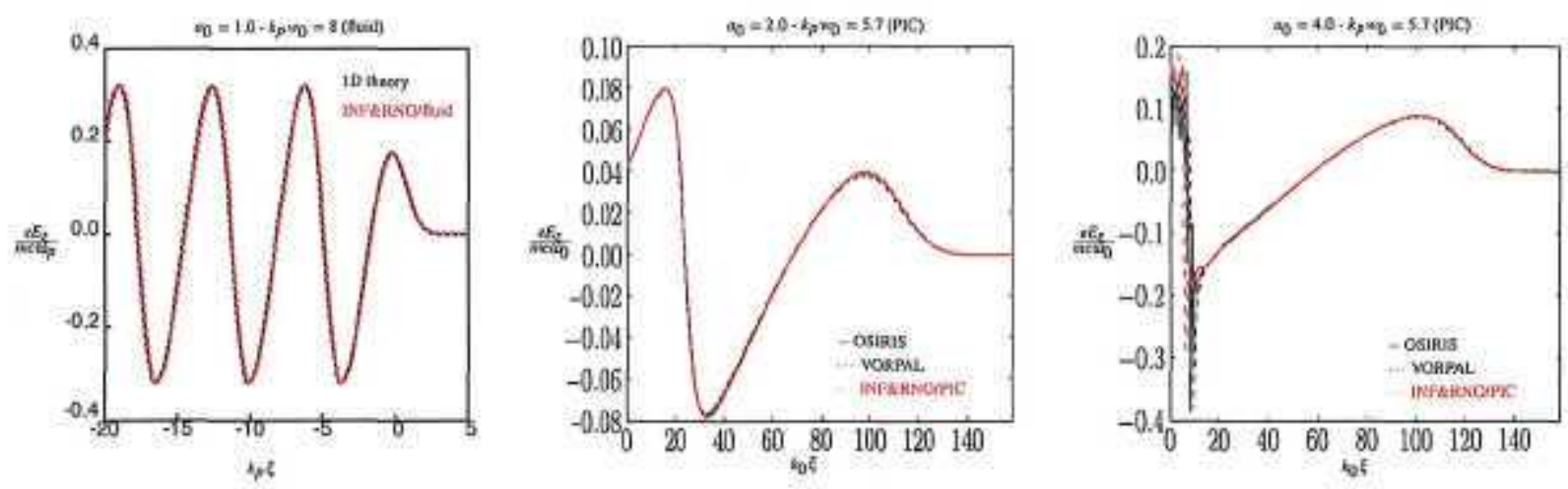

FIGURE 4. Comparison of the longitudinal wakefield obtained using INF\&RNO with the ID quasistatic nonlinear theory (left) and with VORPAL/OSIRIS results [24] (center, right). See subsection 'Test $3^{n}$ for details.

function of the propagation distance: the black curve is the analytic calculation, while the red and blue symbols are simulation results with two different resolutions (see figure). Time integration is performed with RK2. Simulations, even at low resolution, agree with theory. We also checked time reversibility of the simulation. After the forward propagation we evolved back in time the pulse measuring the error in recovering the initial condition. Denoting by $\hat{a}_{i, j}$ the (discretized) initial condition and by $\hat{l}_{h, j}^{\prime}$ the recovered one, the reversibility error can be quantified according to

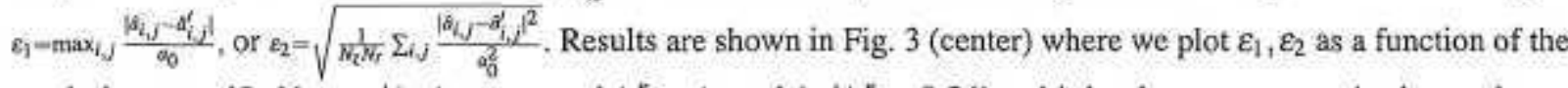
resolution quantified by $w_{0} / \Delta r$ (we assumed $\Delta \xi=\Delta r$ and $\Delta t / \Delta \xi=0.24$ ); a third order convergence is observed.

Test 2 . The accuracy of the laser-plasma coupling has been checked by considering the evolution of a low intensity Gaussian pulse $\left(a_{0}=0.05, k_{p} L=2, k_{p} w_{0}=2.5\right)$ in a plasma channel $\left(k_{0} / k_{p}=20, \Delta n=\Delta n_{c}\right)$. The pulse is slightly mismatched $(\sim 5 \%)$ so we expect amplitude oscillations in the range $[0.05,0.055125]$ with a period of $\omega_{p} T_{\text {osc }}=$ $\pi k_{p} Z_{\text {Rayleigh }}=196.3$. Simulation results showing the time evolution of $a_{0}$ are plot in Fig. 3 (right). The measured oscillation period is 196.6, in good agreement with the theoretical value. The damping of the oscillations, discussed in [21], is due to the fact that the (short) laser pulse is not monochromatic. Each chromatic component of the beam is characterized by a different oscillation frequency and the decoherence between these modes damps out the intensity oscillations. We can eliminate short pulse effects in the simulation removing the mixed derivative $\partial_{\xi, r}^{2}$ in the equation for the envelope evolution (1). Simulation results in this case (black dashed line in Fig. 3 (right)) show no damping and very good agreement with theory.

Test 3. We compare the longitudinal wakefield $\left(E_{z}\right)$ obtained using INF\&RNO with $1 \mathrm{D}$ analytical theory and with other codes (VORPAL[22], OSIRIS[23]) in the nonlinear regime. In Fig. 4 (left) we plot the on-axis lineout of $E_{z}$ generated by a broad Gaussian laser pulse with $a_{0}=1, k_{p} L=2, k_{p} w_{0}=8$. The black plot is the ID quasistatic 


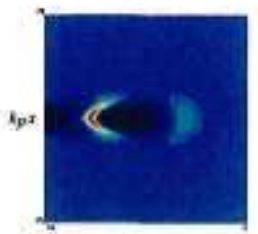

$E_{2}$ (PIC)

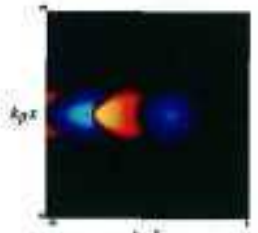

$k 08$

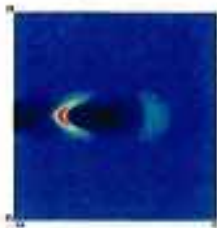

$E_{2}$ (FLUID)



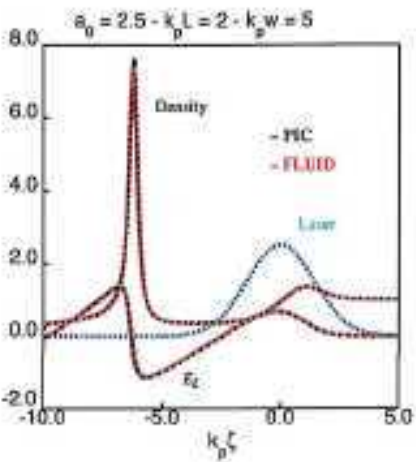

FIGURE 5. Comparison between PIC and fluid modalities in INF\&RNO: snapshots and lineouts for density and longitudinal wakefield (see subsection "Test 4 " for details).

nonlinear theory while the red dashed line is the INF\&RNO/fluid result $\left(k_{p} \Delta \xi=1 / 20, k_{p} \Delta r=1 / 10, \Delta t / \Delta_{2}=0.24\right.$, RK2). The agreement in both phase and amplitude of the wake is very good. In Fig. 4 (center, right) we compare INF\&RNO/PIC with the codes VORPAL and OSIRIS for $a_{0}=2,4$. All the details concerning the benchmarking exercise (laser/plasma parameters, numerical settings for VORPAL and OSIRIS, etc.) can be found in [24]. Concerning INF\&RNO/PIC the simulation has been done with $k_{p} \Delta \xi=1 / 30, k_{p} \Delta r=1 / 10, \Delta t / \Delta \xi=0.24,20$ particles/cell and quadratic shape function. The agreement is excellent for $a_{0}=2$ and good for $a_{0}=4$. We notice that in the $a_{0}=4$ case all the three codes slightly disagree in the rear part of the wake, but this is almost surely due to a numerical convergence issue.

Test 4. In this test we check the internal consistency between the PIC and the fluid modalities of INF\&RNO. For the study we chose to operate in a mildly nonlinear regime where the cold fluid description for the plasma is still valid and, as a consequence, PIC and fluid calculations must give the same answer. The physical parameter for the laser pulse are $a_{0}=2.5, k_{p} L=2, k_{p} w_{0}=5$. For the PIC simulation the numerical parameters are $k_{p} \Delta \xi=1 / 30, k_{p} \Delta r=1 / 20_{t}$ $\Delta t / \Delta \xi=0.24,20$ particles/cell (quadratic shape function). For the fiuid one $k_{p} \Delta \xi=1 / 25, k_{p} \Delta r=1 / 10, \Delta t / \Delta \xi=0.2$. Results are shown in Fig. 5 where we plot snapshots of the electron density and of the longitudinal wakefield from PIC and fluid runs. A comparison of the lineouts along $r=0$ is also shown. Excellent agreement is observed.
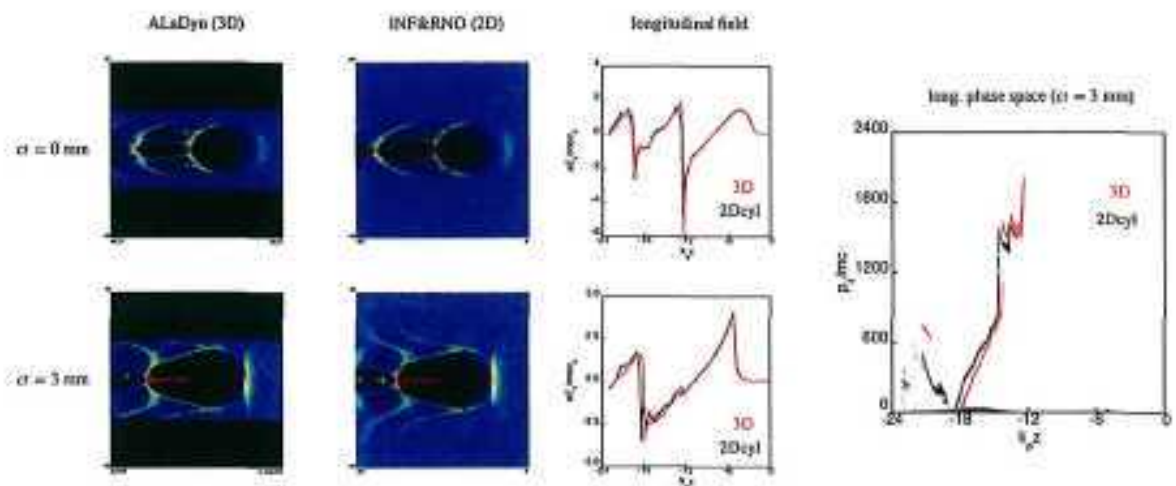

FIGURE 6. Comparison between a full LPA simulation performed with the 3D PIC code ALaDyn and INF\&RNO (see subsection "Test 5 " for details).

Test 5. In the last test presented we compare a full LPA simulation performed with the 3D-PIC code ALaDyn [25, 26] with a run performed with INF\&RNO. Details on the 3D ALaDyn simulation are discussed in [27]: a (Gaussian) laser pulse $\left(a_{0}=5, \tau_{\mathrm{fwhm}}=30 \mathrm{fs}, w_{0}=16 \mu \mathrm{m}\right)$ interacts with a $4 \mathrm{~mm}$ gas-jet (plasma density $3 \cdot 10^{18} \mathrm{e} / \mathrm{cm}^{3}$ ). The numerical parameters for the INF\&RNO simulation are: $k_{p} \Delta \xi=1 / 30, k_{p} \Delta r=1 / 20, \Delta t / \Delta \xi=0.25,6$ particles/cell with particle 
splitting to reduce on-axis nolse and quadratic shape function Results are presented in Fig 6 where we compare the snapshots of the elecrobn density at two different tomes for the two codes, the lneout of the accelerating field and the longitudinal phase space near the end of the simulation The agreement is very good The two codes captore basically the same physics (nonlinear evolution of the laser field, excitation of a bubble-like wake and injection) with the advantage that INF\&RNO is more than 150 times faster compared to the 3D run

\section{CONCLUSION AND OUTLOOK}

We have presented the INF\&RNO computational framework, a 2D cylindrical, envelope, ponderomotive. PIC/fiud simnlation code The code has been whdely tested and some of the benchmarks have been discussed in this paper The plan for improvinghupgrading the code foresees paralleli2ation, enforcement of exact charge conservation, implementation of a quasi-slatic module and introductoon of a boosted Lorentz frame modeling capability Several performance profiling tests confirm a speed-itp of several orders of magintude ( $\sim 2-4$ dependug on the particular probleni) compared to standard simulation tools In particular, in the fiud modality, the CPU time required for one time step has been measured to be $\sim 06 \mu s^{\prime}\left(\right.$ gnd point) In this case, assunung reasonable resolution $\left(k_{p} \Delta \xi \sim 1 / 50, k_{p} \Delta r \sim 1 / 8\right.$, $\left.\mathrm{Nu}_{p} \Delta \sim \sim k_{p} \Delta \xi / 4\right)$ and computatonal domain size $\left(k_{p} L_{2} \sim k_{p} L_{\mathrm{r}} \sim 20-30\right)$, a LPA interaction over a meter-scale plasma ( $g$ g , BELLA) wll take less than 500 CPU hours, makung feasible this knd of runs in a few days on small machnes

\section{ACKNOWLEDGMENTS}

This research is supported by the Department of Energy under the contract No DE-AC02-05CH.1231

\section{REFERENCES}

1 T Tajıma, J M Dawson, Phys Rey Lett 43, p 26] (1979)

2 E Esarey, et al , Rev Mod Phys 81, p 1229 (2009)

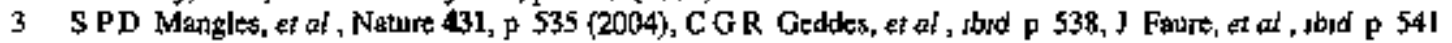

4 W P Leemans, ef al , Nature Physics 2, p 696 (2006)

5 K Nakamura, er ol, Phys Plasmas 14, 05670B (2007)

6 W P Leenans, ef al, these Proceedings

7 C K Bırdsall, A B Langdon, Plasma Physıcs Wia Computer Sımulation, Adarn Hılger. (1991)

8 J -L Way, Phys Rev Lett 98,130405 (2007)

9 S F Martins, ef al, נת Proc 13th Advanced Acoclerator Workehop, Santa Cruz, CA, p 285 (2008)

10 D L Bruhwiler, et al , in Proc 13th Adyanced Accelerator Workshop, Santa Cruz, CA, P 29 (2008)

I1 S F Martins, et at, Nature Physics 6, p 311 (2010)

$12 \mathrm{~J}-\mathrm{L}$ Vay, et al , these Proctedings

13 P Mora, TM Antonsen, Phys Plasmas 4, p 217 (1997)

14 C Huang, et al, I Comp Pbys 217, p $658(2006)$

I5 N H Matlis, ef al, Nature Physucs 2, p $749(2006)$

16 I Blumenfeld, et al , Naiure 445, p 741 (2007)

17 A F Lifshite, ts al, I Counp Phys 228, p 1803 (2009)

18 E Estarey, ef al, Phys Flurds B 5, p 2690 (1993)

19 B A Shadwick, et al , IEEE TPS 30, p 38 (2002)

20 I S Shang, I Comp Phys 153, p 312 (1999)

2I E Esarey, W P Lemang, Phys Rer E 59,p 1052 (1999)

22 C Nieter, JR Cary. J Comp Phys 196, p 448 (2004)

23 R A Hemker, et at, Lecture Notes in Computatianal \$cience 2331, p 342 (2002)

24 K Paul, et at , in Proc 13th Advanced Accelerator Work6hop, Santa Cruz, CA, p 315 (2008)

25 C Benedett, et al , lEEE-TPS 36, p 1790 (2008)

26. C Benedettl, et al, Nuclear Inst and Methods un Physics Research A 6018, p 594 (2L09)

27 C Benedett, an Proc 2nd Conf on Ultrantense Lasct Interaction Sekence, Frascat, Iraky, AlP Conf Proc 1209, p 11 (2010) 


\section{DISCLAIAHER}

This document was prepared as an account of work sponsored by the United States Government. While this document is believed to contain correct information, neither the United States Government nor any agency thereof, nor The Regents of the University of California, nor any of their employees, makes any warranty, express or implied, or assumes any legal responsibility for the accuracy, completeness, or usefulness of any information, apparatus, product, or process disclosed, or represents that its use would not infringe privately owned rights. Reference herein to any specific commercial product process, or service by its trade name, trademark, manufacturer, or otherwise, does not necessarily constitute or imply its endorsement, recommendation, or favoring by the United States Government or any agency thereof, or The Regents of the University of California. The views and opinions of authors expressed herein do not necessarlly state or reflect those of the United States Government or any agency there of or The Regents of the University of California. 